\title{
Martin Luther, personnage de télévision en République fédérale : Le Réformateur (1968) de Günther Sawatzki et Rudolf Jugert
}

Ferdinand Schlie

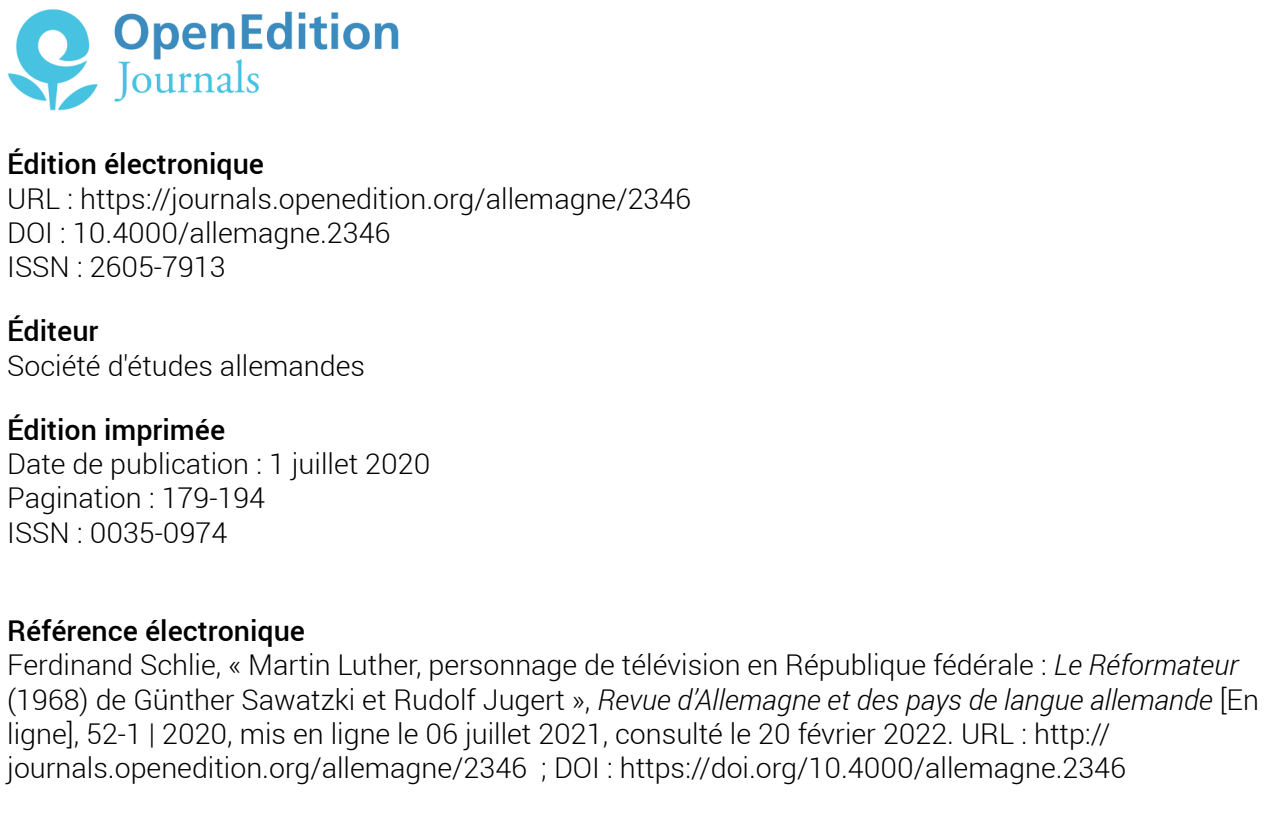




\section{Martin Luther, personnage de télévision en République fédérale: Le Réformateur (1968) de Günther Sawatzki et Rudolf Jugert}

\section{- Ferdinand Schlie*}

Outre-Rhin, 2017 fut incontestablement l'«année Luther». À l'occasion du $500^{\mathrm{e}}$ anniversaire des 95 thèses, fortement centré sur la personne du Réformateur, la commémoration prit les formes les plus diverses, allant du colloque scientifique à la comédie musicale, du jeu vidéo au canard de bain à l'effigie du moine rebelle ${ }^{(1)}$. Parmi les supports employés pour entretenir la mémoire de Luther, le film de télévision ne fut pas en reste et proposa des approches partiellement novatrices du Réformateur. Katharina Luther de Julia von Heinz fut le premier film de fiction entièrement centré sur l'épouse de Luther; voué à « décentrer» le jubilé de la personne du Réformateur, il sensibilisa le public au "chemin d'une libération » ${ }^{(2)}$ parcouru par une femme à l'aube de l'époque moderne. Entre le ciel et l'enfer (Zwischen Himmel und Hölle) d'Uwe Janson insista sur le rôle joué par Andreas Karlstadt et Thomas Müntzer durant les débuts de la Réforme, tendant ainsi à présenter celle-ci comme un «travail d'équipe» ${ }^{(3)}$.

Il est moins connu - notamment en France - que ces productions récentes s'inscrivent dans une longue tradition dont les premiers exemples remontent à l'époque wilhelmienne. Corpus d'étude des plus passionnants ${ }^{(4)}$, les «Lutherfilme» documentent

* Docteur en études germaniques, ancien élève de l'ENS de Lyon, professeur agrégé d'allemand au lycée Georges Cuvier à Montbéliard.

1 Voir entre autres Benjamin HasselHorn (éd.), Luther vermitteln. Reformationsgeschichte zwischen Historisierung und Aktualisierung, Leipzig, Evangelische Verlagsanstalt, 2016.

2 «der Weg einer Befreiung», Mario KReBs, «"Katharina”. Spielfilm für die ARD», in: B. HAsselHORN (éd.), Luther vermitteln (note 1), p. 229-234, cit. p. 234.

3 «Teamleistung», Oliver Jungen, «Die Saat des Gewissens», Frankfurter Allgemeine Zeitung, 30.10.2017, www.faz.net/aktuell/feuilleton/medien/tv-kritik/der-luther-film-im-zdf-zwischen-himmelund-hoelle-15266500.html (consulté le 31.08.2018).

4 Pour des analyses approfondies qui tentent de saisir le phénomène sur de longues périodes, voir Esther P. Wipfler, Martin Luther in Motion Pictures. History of a Metamorphosis, Göttingen, Vandenhoeck 
sur plus d'un siècle d'histoire allemande les tentatives de mobiliser la figure du Réformateur pour à la fois diffuser largement, par le biais d'un média populaire, des représentations préexistantes, et façonner activement l'image de Luther dans la mémoire collective. Prises dans une oscillation entre la «mythisation» du Réformateur - c'està-dire son établissement en figure fondatrice et exemplaire à partir d'un canevas d'épisodes biographiques connus, reconfigurés selon les besoins du moment ${ }^{(5)}$ - et la «démythisation» de ce même personnage, les productions filmiques font pour ainsi dire office de baromètre qui reflète les questionnements liés à l'identité collective ${ }^{(6)}$ notamment à l'échelle nationale et confessionnelle.

Particulièrement nombreuses, les productions télévisées allemandes de l'aprèsguerre ont joui au cours des quinze dernières années d'un regain d'intérêt ${ }^{(7)}$ mais n'ont pas encore reçu au sein des publications existantes toute l'attention qu'elles méritent. C'est à l'étude plus détaillée de ce pan du corpus que le présent article cherche à

\& Ruprecht, 2011; Ferdinand Schlie, Luther à l'écran. La figure du Réformateur au cinéma et à la télévision en Allemagne (1911-2008), thèse sous la direction de François Genton, soutenue à l'Université Grenoble Alpes le 7 décembre 2018 (nous citons la version de diffusion de la thèse, déposée aux Bibliothèques universitaires de Grenoble).

5 Sur la notion de mythe, voir Karin Bruns, Rolf PARr, Wulf Wülfing, Historische Mythologie der Deutschen, Munich, Fink, 1991, p. 1-17; Herfried Münkler, Die Deutschen und ihre Mythen, Hambourg/Reinbek, Rowohlt Taschenbuch, 2010, p. 9-30; Jan Assmann, La mémoire culturelle. Écriture, souvenir et imaginaire politique dans les civilisations antiques, trad. par Diane Meur, Paris, Aubier, 2010, p. 47 et p. 71-75.

6 Sur ce concept, voir J. Assmann, La mémoire culturelle (note 5), p. 119.

7 Esther P. Wipfler, «Vom deutschnationalen Titan zum Herzensbrecher. Neunzig Jahre Luther-Film. Zur Geschichte des Luther-Bildes in Kinematographie und Fernsehen ", Luther. Zeitschrift der Luthergesellschaft, $\mathrm{n}^{\circ} 75$ (2004), p. 25-41; ID., Martin Luther in Motion Pictures (note 4), p. 17-19, p. 29-35, p. 51-64 et p. 72-77; ID., «Filmstar Martin Luther. Projektionen einer Kult-Figur», in: Hole RössLER (éd.), Luthermania. Ansichten einer Kultfigur, catalogue d'exposition, Wolfenbüttel, Herzog August Bibliothek (15.01.2017-17.04.2017), Wiesbaden, Harrassowitz, 2017, p. 91-100; Hans-Rüdiger ScHwA B, "Luther im deutschen Fernsehen. Signale an ein Massenpublikum », in: B. HasselHorn (éd.), Luther vermitteln (note 1), p. 208-228; Markus Pohlmeyer, "Martin Luther (1983): Filmische Rekonstruktion eines Genies. Und eine Demontage. Ein Essay», in: Günter Helmes (éd.), "Schicht um Schicht behutsam freilegen». Die Regiearbeiten von Rainer Wolffhardt, Hambourg, Igel, 2012, p. 278288; Stewart Anderson, "Martin Luther in Primetime. Television Fiction and Cultural Memory Construction in Cold War Germany», Journal of European Television History \& Culture, $\mathrm{n}^{\circ} 3$ (2012), p. 22-26; Ferdinand Schlie, «Le Réformateur dédoublé: Martin Luther à l'écran dans les "deux Allemagnes" en 1983 ", Chrétiens et Sociétés XVI -XXI $^{e}$ siècles, $\mathrm{n}^{\circ} 23$ (2016): Les anniversaires de la Réforme, dossier coordonné par Yves Krumenacker, p. 93-115. Pour des travaux plus anciens, voir Rotraut Simons, «Das DDR-Fernsehen und die Luther-Ehrung», in: Horst DÄHN, Joachim HeIse (éd.), Luther und die DDR. Der Reformator und das DDR-Fernsehen 1983, Berlin, Edition Ost, 1996, p. 99-185; Fritz Hufen, Margaret Tra pmann (éd.), Martin Luther. Reformator - Ketzer - Nationalheld? Texte, Bilder, Dokumente in ARD und ZDF. Materialien zu Fernsehsendungen, Munich, Goldmann, 1983; Johannes Horstmann (éd.), Martin Luther. Zum Wandel des Luther-Bildes in der Geschichtsschreibung und im Film, Schwerte, Katholische Akademie Schwerte, 1983; Hans Süssmuth, «Luther im Zugriff des Fernsehens. Zwischen kritischer Deutung und Vermittlung traditioneller Klischees», in: ID. (éd.), Das Luther-Erbe in Deutschland. Vermittlung zwischen Wissenschaft und Öffentlichkeit, Düsseldorf, Droste, 1985, p. 337-361; Hans Joachim DörGER, «Schlecht vorbereitet - lieblos arrangiert. Luther in den Medien. Analyse der Fernsehproduktionen», in: Claus-Jürgen Roepke (éd.), Luther 83. Eine kritische Bilanz, Munich, Christian Kaiser, 1984, p. 97-111. 
contribuer en se consacrant à un exemple précis, pour lequel le constat d'une lacune de la recherche est particulièrement frappant ${ }^{(8)}$. Diffusé par la seconde chaîne allemande (Zweites Deutsches Fernsehen, ZDF) en 1968, Le Réformateur (Der Reformator), réalisé par Rudolf Jugert d'après un scénario de Günther Sawatzki, n'a fait à ce jour l'objet d'aucune publication conséquente ${ }^{(9)}$ alors qu'il témoigne d'un moment charnière dans la réception audiovisuelle de Luther en Allemagne, marqué par la reconsidération en profondeur d'un mythe caractérisé au moins jusqu'en 1945 par un triomphalisme et/ou un revanchisme nationaliste et protestant.

L'objectif de cette étude est de saisir à la fois les motifs qui guidèrent cette production dans la République fédérale des années 1960 et les ressorts narratifs et visuels mis en œuvre pour véhiculer une image renouvelée de Martin Luther. Un premier temps de réflexion retracera les évolutions que subit le discours sur le Réformateur dans l'Allemagne de l'Ouest au lendemain de la guerre, précisera les enjeux liés au média télévisuel et reconstituera l'élaboration du scénario à partir de sources d'archives. Dans un deuxième temps, il s'agira de dégager les principaux aspects de la caractérisation de Luther véhiculée par le film. En guise de conclusion, nous retracerons l'accueil qui fut réservé à cette production à la fois par les téléspectateurs et la presse ouest-allemande.

\section{Un scénario et son contexte}

$\mathrm{Au}$ lendemain de la Seconde Guerre mondiale, les évolutions qui affectèrent la société allemande vinrent affaiblir, voire porter un coup fatal aux éléments ayant alimenté jusqu'ici le mythe qui s'était constitué autour de la figure de Martin Luther. La "césure fondamentale» ${ }^{(10)}$ que représente la Seconde Guerre mondiale dans l'histoire du nationalisme allemand est un premier facteur à prendre en compte. Exacerbé par le nazisme, le culte de la nation avait mené le pays à la catastrophe; une "profonde désillusion » ${ }^{(11)}$ alla de pair avec une "perte de légitimité du nationalisme » ${ }^{(12)}$ qui avait constitué jusqu'ici un ressort essentiel du mythe de Luther et d'autres figures analogues, érigées en 1871 en figures de proue du Reich nouvellement fondé. Gardien non seulement de la nation mais aussi de la foi protestante, Martin Luther fut également victime d'un double phénomène ayant trait à la place de la religion dans la société

8 Le présent article s'appuie sur les recherches que nous avons menées dans le cadre de notre thèse de doctorat; il reprend notamment, de manière abrégée et légèrement remaniée, les principaux éléments et passages des chapitres 13 et 15 de celle-ci. Voir F. Schlie, Luther à l'écran (note 4), p. 359-371 et p. 388-407. Nos analyses sont menées à partir de la version DVD du film qui nous a été fournie par les archives de la Zweites Deutsches Fernsehen.

9 Pour des premiers éléments de réflexion, voir J. Horstmann (éd.), Martin Luther (note 7), p. 109-115; E. Wipfler, «Vom deutschnationalen Titan» (note 7), p. 35; ID., Martin Luther in Motion Pictures (note 4), p. 53; H.-R. Sсншав, «Luther im deutschen Fernsehen» (note 7), p. 214-215.

10 «fundamentale Zäsur», Hans-Ulrich WeHLER, Nationalismus. Geschichte-Formen - Folgen, Munich, C.H. Beck, 2011, p. 87.

11 «tiefe Desillusionierung», ibid.

12 «Entlegitimierung des [...] Nationalismus», Heinrich August WinkLer, «Nationalismus, Nationalstaat und nationale Frage in Deutschland seit 1945 », in: Hartmut Kaelble, Heinrich August Winkler (éd.), Nationalismus - Nationalitäten - Supranationalität, Stuttgart, Klett-Cotta, 1993, p. 12-33, cit. p. 12 . 
allemande, à savoir une "accélération du processus de sécularisation " ${ }^{(13)}$ qui gagna l'Allemagne et l'Europe dans la seconde moitié du $\mathrm{XX}^{\mathrm{e}}$ siècle, et l'estompement des différences traditionnelles entre les confessions ${ }^{(14)}$.

L'effritement des paradigmes qui avaient exercé jusqu'ici une influence majeure sur l'image de Luther en Allemagne brisa la dominance d'un discours hagiographique sur le Réformateur et rendit possible l'émergence et la diffusion progressives de nouveaux accès au personnage. Le jubilé de 1946 qui commémora les 400 ans de la mort de Luther en fut un premier indicateur; «célébr[é] en demi-teinte » ${ }^{(15)}$, l'anniversaire fut l'occasion de poser une question brûlante: "Luther était-il l'ancêtre spirituel d'Hitler? " ${ }^{(16)}$. Il convient toutefois de constater que les points de vue qui soulignaient dans l'immédiat après-guerre l'héritage problématique du Réformateur restèrent trop marginaux pour déconstruire durablement l'image du héros national ${ }^{(17)}$, constat également valable pour le jubilé en question ${ }^{(18)}$.

Ce fut notamment de la recherche universitaire que vint à partir du début des années 1960 une nouvelle conception de Luther, introduite par une «expérimentation avec de nouvelles méthodologies "post-confessionnelles" " ${ }^{(19)}$. Les historiens de l'Église Bernd Moeller et Heiko Oberman développèrent des «approches de recherche qui situaient Luther, dont l'originalité et l'exceptionnalité théologiques avaient été mises en évidence durant des décennies [...], au sein d'une conception plus large du cours de l'histoire et le replacèrent dans le contexte de la fin du Moyen Âge et des Réformateurs qui avaient existé à côté de lui » ${ }^{(20)}$. S'inspirant de cette démarche, les historiens développèrent à l'échelle internationale un vif intérêt pour l'époque de la Réforme qui intégra «les contextes sociaux du développement et de la réception de la théologie réformatrice ${ }^{(21)}$. Plus spécifiquement consacrés au Réformateur, d'autres travaux de recherche reconsidérèrent à la lumière des sources certains «grands moments » de la biographie

13 «Beschleunigung des Säkularisierungsprozesses», Edgar Wolfrum, Die geglückte Demokratie. Geschichte der Bundesrepublik Deutschland von ihren Anfängen bis zur Gegenwart, Stuttgart, KlettCotta, 2006, p. 75.

14 Gérald Chaix, «La Réformation», in: Étienne François, Hagen Schulze (éd.), Mémoires allemandes, Paris, Gallimard, 2007, p. 91-119, voir p. 113.

15 Ibid.

16 «War Luther Hitlers geistiger Ahnherr?», Johannes Schilling, «Luther 1946 », in: Jan SCHEUNEMANN (éd.), Reformation und Bauernkrieg. Erinnerungskultur und Geschichtspolitik im geteilten Deutschland, Leipzig, Evangelische Verlagsanstalt, 2010, p. 183-195, cit. p. 189.

17 Hartmut Lehmann, «Katastrophe und Kontinuität. Die Diskussion über Martin Luthers historische Bedeutung in den ersten Jahren nach dem Zweiten Weltkrieg», in: ID., Luthergedächtnis 1817 bis 2017, Göttingen, Vandenhoeck \& Ruprecht, 2012, p. 189-212, voir p. 209-210.

18 Ibid., p. 193-195.

19 "Experimentation with new, "post-confessional" methodologies», Thomas A. HowARD, Remembering the Reformation. An Inquiry into the Meaning of Protestantism, Oxford, Oxford University Press, 2016, p. 125.

20 «Forschungsansätze [...], die Luther, dessen theologische Originalität und Exzeptionalität jahrzehntelang [...] herausgearbeitet worden war, in einen breiteren Strom der Geschichte einordneten und in Bezug auf das späte Mittelalter und die Reformatoren neben ihm kontextualisierten ", Thomas Kaufmann, «Die deutsche Reformationsforschung seit dem Zweiten Weltkrieg», Archiv für Reformationsgeschichte, $\mathrm{n}^{\circ} 100$ (2009), p. 15-47, cit. p. 29.

21 «die sozialen Kontexte der Ausbildung und Rezeption reformatorischer Theologie», ibid. 
de Luther, contribuant ainsi à lui faire perdre son «aura de héros national» ${ }^{(22)}$. En complément des impulsions novatrices venues d'Allemagne, un regard nouveau fut posé outre-Atlantique sur le Réformateur: le psychanalyste germano-américain Erik H. Erikson publia en 1958 l'étude Le jeune homme Luther ${ }^{(23)}$, ouvrage contestable et contesté( $^{(24)}$ qui voit à l'œuvre dans le cheminement intellectuel du Réformateur des mécanismes inconscients.

Si la fin de la Seconde Guerre mondiale marque le début d'une rupture profonde avec un investissement héroïque du mythe de Luther, la volonté de déconstruire cette figure gigantesque ne constitua pas la seule tendance des deux décennies qui suivirent; elle entra au contraire en tension avec d'autres approches qui renouèrent avec une image positive de Luther. Parmi les œuvres littéraires qui lui furent consacrées, certaines témoignent d'une actualisation du mythe qui perpétue les lectures hagiographiques d'avant-guerre ${ }^{(25)}$. Certaines productions filmiques des années 1950 s'inscrivent tout autant dans l'optique d'une «hagiographie de Luther » (26), à l'image du film documentaire Le rebelle obéissant (Der gehorsame Rebell) de Curt Oertel (1952) ${ }^{(27)}$. Au-delà de ces lectures traditionnelles, on constate également un réinvestissement du mythe sous de nouveaux auspices: Thomas A. Howard note ainsi qu'au sein du bloc occidental, la présence d'un «courant sous-jacent» qui voyait dans la Réforme «les germes de la démocratie libérale, de la tolérance et des temps modernes» venait contrebalancer le "pluralisme» ${ }^{(28)}$ des interprétations. On en trouve un écho dans le film biographique Martin Luther du réalisateur américain Irving Pichel, sorti en salle en Allemagne en 1954 et qui tend à insister sur la dimension exemplaire que le Réformateur pouvait revêtir sur le plan politique dans le contexte de l'après-guerre: la «résist[ance]» contre un «abus arbitraire du pouvoir» ${ }^{(29)}$, la promotion de la "démocratie au lieu de la hiérarchie et de la monarchie » ${ }^{(30)}$ s'y trouvent accentuées, risquant ainsi de placer au second plan la dimension religieuse de la démarche de Luther au profit d'une "émancipation séculière et moderne ${ }^{(31)}$.

22 «Nimbus als Nationalheld», H. LehmanN, «Katastrophe und Kontinuität» (note 17), p. 211.

23 Erik H. Erikson, Young Man Luther. A Study in Psychoanalysis and History, New York, Norton, 1958.

24 Voir par exemple Heinz Schilling, Martin Luther. Rebell in einer Zeit des Umbruchs. Eine Biographie, Munich, C. H. Beck, 2013, p. 63-64.

25 Voir Norbert Mecklenburg, Der Prophet der Deutschen. Martin Luther im Spiegel der Literatur, Stuttgart, Metzler, 2016, p. 228; Michael GöRING, "Luther und die Reformation als Gegenstand des historischen Dramas der Gegenwart», Literaturwissenschaftliches Jahrbuch, $\mathrm{n}^{\circ} 28$ (1987), p. 263-282, en particulier p. 278-279.

26 «Luther-Hagiographie», François Traudisch, «Das Lutherbild in deutschen Filmen », in: J. HorstMANN (éd.), Martin Luther (note 7), p. 37-45, cit. p. 43.

27 Werner Sснмідт-QuindeAu, «Der Reformator als Leinwandheld: Lutherfilme zwischen Geschichte und Ideologie», in: Thomas Bohrmann, Werner Veith, Stephan Zöller (éd.), Handbuch Theologie und populärer Film, t. 2, Paderborn/Munich/Vienne/Zurich, Schöningh, 2009, p. 189-194, voir p. 191-192.

28 "undercurrent», "the seeds of liberal democracy, tolerance, and the modern age», "pluralism», T. HowARD, Remembering the Reformation (note 19), p. 122.

29 «resist arbitrary abuse of power», E. WIPFLER, Martin Luther in Motion Pictures (note 4), p. 49.

30 «democracy instead of hierarchy and monarchy», ibid.

31 «modern-säkulare Emanzipation», F. TrAUdisch, «Das Lutherbild» (note 26), p. 43. 
Diffusée par la Zweites Deutsches Fernsehen, la production analysée ici reflète doublement les nouvelles approches du Réformateur que nous avons évoquées. D’une part, elle offre au public un portrait de Luther largement débarrassé du monumentalisme qui avait longtemps dominé le discours sur l'auteur des 95 thèses; elle véhicule cette approche par le biais d'un scénario dense, exigeant, parfois même difficile d'accès, sollicitant la concentration et la réflexion ${ }^{(32)}$. De l'autre, elle procède à un réinvestissement prudent du mythe de Luther, destiné cette fois à contribuer à la construction d'une République fédérale démocratique et pluraliste. Cette double caractéristique découle à la fois du rôle dévolu à la télévision publique en RFA et des enjeux spécifiques liés à la "pièce documentaire» («Dokumentarspiel»), genre dans lequel s’inscrit Le Réformateur. Esther P. Wipfler a raison de rappeler la dimension «éducative» ${ }^{(33)}$ caractéristique de la télévision publique dans la République fédérale des années 1960: cette décennie vit en effet l'émergence d'un paradigme accentuant la vocation de la télévision à être vecteur à la fois de connaissances, d'orientation pratique face aux défis de l'existence et de formation à l'esprit critique ${ }^{(34)}$. On mit en évidence la dimension pédagogique d'un moyen de communication désormais accessible à la grande majorité de la population dans tous les milieux sociaux ${ }^{(35)}$.

À ce premier enjeu s'en ajoute un autre qui y est étroitement lié, à savoir la valorisation de la télévision face aux critiques dont celle-ci faisait l'objet. C'est là un aspect déjà pointé par Hans-Rüdiger Schwab, qui rappelle que le téléfilm de 1968 s'inscrit dans un contexte où bon nombre d'intellectuels reprochaient à la télévision, ce «média de masse hautement suspect ${ }^{(36)}$, d'abêtir et d'uniformiser le public plutôt que de favoriser l'émergence d'une pensée autonome et singulière.

Nourrie par les considérations développées jusqu'ici, l'élaboration du film Le Réformateur visait plus précisément à respecter les codes de la "pièce documentaire", genre qui reposait sur le principe de la «reconstruction scénique d'une période déterminée du passé » ${ }^{(37)}$ à partir de "textes originaux» et de «matériel iconographique de l'époque concernée " ${ }^{(38)}$. Un ou plusieurs auteurs adaptaient la matière ainsi accumulée pour l'écran en élaborant un scénario conforme aux «règles de la dramaturgie conventionnelle» ${ }^{(39)}$, fondé sur une "action achevée et cohérente» ${ }^{(40)}$ que ponctuaient des «sommets dramatiques» ${ }^{(41)}$. Les productions ainsi obtenues

32 Voir également E. Wipfler, Martin Luther in Motion Pictures (note 4), p. 53.

33 «educational», ibid.

34 Knut Hickethier, Geschichte des deutschen Fernsehens, Stuttgart/Weimar, Metzler, 1998, p. 220-221 et p. 227.

35 Ibid., p. 200.

36 «höchst verdächtige[s] Massenmedium», H.-R. ScHwab, «Luther im deutschen Fernsehen» (note 7), p. 211.

37 «szenische[-] Rekonstruktion eines ausgewählten Zeitabschnittes der Vergangenheit», Knut Hicket hier, Das Fernsehspiel der Bundesrepublik. Themen, Form, Strukturen, Theorie und Geschichte, 1951-1977, Stuttgart, Metzler, 1980, p. 283.

38 «Originaltexte», «Bildmaterial der betreffenden Zeit», ibid.

39 «Regeln konventioneller Dramaturgie», ibid.

40 "in sich abgeschlossene Handlung», ibid., p. 283-284.

41 «dramatische[-] Höhepunkte[-]», ibid., p. 284. 
renfermaient une visée doublement didactique: d'une part, elles cherchaient à apporter par le biais d'un récit plaisant des connaissances historiques exactes ${ }^{(42)}$; de l'autre, elles étaient conçues selon les termes de Wolfgang Bruhn, directeur de l'unité chargée des pièces documentaires à la ZDF de 1965 à 1969, comme un vecteur $\mathrm{d}^{\prime}$ "éducation à la citoyenneté et de conscience citoyenne»" ${ }^{(43)}$ dans la mesure où elles présentaient au spectateur des personnages modèles incarnant des principes moraux jugés positifs ${ }^{(44)}$, principes constitués dans le cas de Luther par l'esprit critique et l'autonomie intellectuelle.

Les rappels d'ordre général effectués jusqu'ici éclairent de manière évidente les enjeux liés au film Le Réformateur, qu'il convient de préciser désormais en retraçant l'histoire de son scénario. À ce titre, la personnalité du scénariste Günther Sawatzki mérite une attention particulière. Les textes publiés par Sawatzki après la Seconde Guerre mondiale font état d'un souci récurrent, à savoir la préservation des libertés de l'individu face aux instances de tous types susceptibles de la brider. Cette position est d'autant plus remarquable qu'elle forme un contraste évident avec les débuts du parcours de Sawatzki, marqués encore par des propos qui évoquent l'idéologie national-socialiste ${ }^{(45)}$. Pendant la guerre, Sawatzki s'était essayé comme critique de cinéma, saluant les productions qui célébraient la puissance de la flotte et de l'aviation allemandes ${ }^{(46)}$ ou mettaient en scène, tel Le Grand roi de Veit Harlan, la «fermeté héroïque " d'un individu "téméraire et génial» ${ }^{(47)}$.

Le repositionnement idéologique qu'opéra Sawatzki au lendemain de la guerre lui permit de s'insérer dans un paysage médiatique visant désormais à reconstruire le pays sur des fondements démocratiques. Poursuivant sa carrière de journaliste, il travailla au quotidien Die Welt de 1946 à $1950^{(48)}$ et à la Nordwestdeutscher Rundfunk durant les cinq années qui suivirent ${ }^{(49)}$ avant d'être nommé rédacteur en chef du Kölner

42 Christian Hissnauer, «Geschichtsspiele im Fernsehen. Das Dokumentarspiel als Form des hybriden Histotainments der 1960er und 1970er Jahre», in: Klaus Arnold, Walter Hömberg, Susanne KinNEBRock (éd.), Geschichtsjournalismus. Zwischen Information und Inszenierung, Berlin/Münster, Lit, 2010, p. 293-316, voir p. 293.

43 «staatsbürgerliche Bildung und Staatsbürgerbewußtsein», Wolfgang BruHN, «Die Illusion des Authentischen. Definition und Dramaturgie des Fernsehspiels», in: Christian Longolius (éd.), Fernsehen in Deutschland. Gesellschaftspolitische Aufgaben und Wirkungen eines Mediums, Mayence, v. Hase \& Koehler, 1967, p. 157-163, cit. p. 159.

44 K. Hickethier, Das Fernsehspiel (note 37), p. 285.

45 Voir Günther Sawatzki, «Das neue Feuilleton », Die Literatur, nº 36 (1933/1934), p. 72-73. Cité d'après Joseph Wulf, Presse und Funk im Dritten Reich. Eine Dokumentation, Berlin/Francfort-sur-le-Main/ Vienne, Ullstein, 1983, p. 215-216.

46 Rolf Giesen, Manfred Новsch, Hitlerjunge Quex, Jud Süss und Kolberg. Die Propagandafilme des Dritten Reiches. Dokumente und Materialien zum NS-Film, Berlin, Schwarzkopf \& Schwarzkopf, 2005, p. 319 et p. 333.

47 «heroische Standhaftigkeit», «kühn und genial», Günther SAwATzki, «Das Herz von Stahl. Zu dem Film "Der große König”", Berliner Lokal-Anzeiger (Unterhaltungsbeilage), 28.02.1942.

48 Stefan Andres, Günther Sawatzki, Wilhelm Treue, Jahr und Jahrgang 1906, Hambourg, Hoffmann \& Campe, 1966, p. 165.

49 Ibid.; Herbert A. Frenzel, Hans Joachim Moser, Kürschners Biographisches Theater-Handbuch, Berlin, De Gruyter, 1956, p. 633. 
Stadt-Anzeiger de 1956 à $1961^{(50)}$. Défenseur des «personnes qui, de manière démocratique, souhaitent se former un jugement par elles-mêmes » ${ }^{(51)}$, Sawatzki s'en prit aux autorités qui, mises à mal par le nazisme, cherchaient selon lui à rétablir petit à petit leur emprise ${ }^{(52)}$; il appelait à la vigilance, insistant notamment sur le rôle de l'œuvre d'art comme remède à l'endormissement et à l'uniformisation de la société( ${ }^{(53)}$.

Les mises en garde de Sawatzki font apparaître en creux l'idéal d'un individu autonome, doté d'un esprit critique, singulier dans sa manière de penser et de percevoir. Aussi peut-on voir dans les contributions de Sawatzki à plusieurs productions télévisées des années 1960 et 1970 une tentative d'amener le téléspectateur vers l'idéal qui sous-tendait ses textes. Consacrées pour trois d'entre elles à un épisode de l'histoire allemande ${ }^{(54)}$, les productions auxquelles collabora Sawatzki partagent une approche du passé reposant sur l'étude des sources et la maîtrise des travaux de recherche menés sur le sujet; aux représentations de l'histoire fondées sur des idées reçues, il entendait opposer une autre manière d'aborder le passé, caractérisée par une recherche des faits établis et une interprétation éclairée par les études de spécialistes.

C’est précisément de la volonté de rompre avec les représentations hagiographiques et convenues de Martin Luther que témoigne l'élaboration du scénario du film Le Réformateur, documentée par les archives de la ZDF (ZDF-Unternehmensarchiv) à Mayence. En effet, on entendit à la fois fonder l'entreprise sur des données fiables ${ }^{(55)}$ et inclure dans le récit des éléments biographiques peu propices à nourrir l'image simpliste du héros sans faille.

Lors d'un premier entretien ${ }^{(56)}$, Günther Sawatzki et Bert Bieniek, chargé de programmes (Redakteur) responsable du projet à la ZDF, fixèrent le cadre du travail à venir en déterminant un ensemble de 38 scènes que contiendrait le scénario et qui couvraient la période comprise entre les 95 thèses et la confession d'Augsbourg. À partir de ce canevas, Sawatzki élabora une esquisse détaillée des scènes retenues accompagnée de commentaires révélateurs ${ }^{(57)}$. Le scénariste souligna ainsi la nécessité

50 Andres/Sawatzki/Treue, Jahr und Jahrgang 1906 (note 48), p. 165; Kurt Weinhold, Die Geschichte eines Zeitungshauses: 1620-1945. Eine Chronik 1945-1970, Cologne, Du Mont Schauberg, 1969, p. 302 et p. 304. Voir également, Hans Schмıтz, Kölner Stadt-Anzeiger. Das Comeback einer Zeitung 19491989, Cologne, DuMont, 1989, p. 40 et p. 42.

51 «Leute [...], die sich, nach demokratischer Art, ihr Urteil selbst zu bilden wünschen», Günther SAWATZKI, "Tagesprobleme des Hörspiels», Rundfunk und Fernsehen, nº 4 (1954), p. 135-145, cit. p. 145.

52 Ibid., p. 141.

53 Ibid., p. 142-143.

54 Outre le film étudié ici, il s'agit des productions suivantes: Ludwig Cremer (réal.), La fuite en Hollande (Die Flucht nach Holland), 1967; Rudolf Jugert (réal.), La Prusse par-dessus tout - L'unification allemande de Bismarck (Preußen über alles - Bismarcks deutsche Einigung), 1970.

55 Le souci d'objectivité et de factualité dont témoigne le film est également relevé par E. WIPFLER, Martin Luther in Motion Pictures (note 4), p. 53 et H.-R. ScHwAB, «Luther im deutschen Fernsehen» (note 7), p. 214-215.

56 Bert Bieniek, «Protokoll über die Besprechung mit Herrn Dr. Sawatzki am 09.10.1967 zu dem Dokumentarspiel "Martin Luther" ", 10.11.1967, ZDF-Unternehmensarchiv, "Produktionsakte Der Reformator (Prod.-Nr. 06361/00119)».

57 Courrier de Günther SAwATZKi à Wolfgang BRuhn, 31.01.1968, ZDF-Unternehmensarchiv, "Produktionsakte Der Reformator (Prod.-Nr. 06361/00119)». 
de s'attarder sur les années 1517-1521 non pour célébrer les débuts héroïques de la rupture avec la hiérarchie ecclésiastique mais pour insister au contraire sur le fait que le moine «se détach[a] malgré lui de l'Église, de l'autorité papale » ${ }^{(58)}$. S’il fallait rendre compte de sa «dureté éclatante», il s'agissait également de ne pas taire ses «doutes » ${ }^{(59)}$. Quant aux événements postérieurs à 1521, Sawatzki mit en avant le rôle des "grands collaborateurs de Luther ${ }^{(60)}$, évitant ainsi de représenter la Réforme comme l'œuvre d'un seul homme. Dernier point notable, le scénariste nota que Luther perdit le contrôle du mouvement qu'il avait lui-même instigué et qu'il s'agissait de rendre compte de la «résignation» ${ }^{(61)}$ qui s'empara de lui. Les courriers ultérieurs de Sawatzki témoignent de l'effort de véhiculer ce portrait nuancé de Luther par le biais d'un scénario à la fois bien renseigné et apte à capter l'attention du public ${ }^{(62)}$.

Sensible à l'évolution des rapports interconfessionnels, Bert Bieniek nota la nécessité de garantir l'exactitude historique du scénario et de prévenir ainsi d'éventuelles objections du côté catholique. À cette fin, il conseilla la consultation de deux spécialistes qui représenteraient chacun l'une des deux grandes confessions ${ }^{(63)}$. Gottfried Edel, chargé de programmes au sein de l'unité «culture» à la ZDF, protestant et «expert de Luther ${ }^{(64)}$ selon Bieniek, assura la première expertise et émit un avis favorable ${ }^{(65)}$. On envisagea de faire appel pour la deuxième expertise à Joseph Lortz, éminent spécialiste de la Réforme qui avait fortement contribué à modifier le regard porté sur Luther par la recherche catholique; Lortz semble toutefois avoir été remplacé par Elmar Lorey, chargé de programmes au sein de l'unité «Église et vie» («Kirche und Leben») à la ZDF, qui donna lui aussi son feu vert ${ }^{(66)}$. Dernier obstacle à franchir, on dut raccourcir le scénario ${ }^{(67)}$ afin de ne pas dépasser la durée impartie par Wolfgang Bruhn, directeur de l'unité "pièces documentaires" à la ZDF; plutôt que de supprimer des scènes entières, on décida de raccourcir plusieurs d'entre elles, créant ainsi une plus grande densité au sein du scénario plutôt que d'éliminer intégralement les aspects couverts par les scènes en question ${ }^{(68)}$.

\footnotetext{
58 «sich [...] widerwillig von der Kirche, der päpstlichen Autorität löst», ibid.

59 «blitzende Härte», «Zweifel», ibid.

60 «grosse[-] [sic] Mitarbeiter Luthers», ibid.

61 «Resignation», ibid.

62 Courriers de Günther SAwatzki à Bert Bieniek, 14.03.1968 et 17.03.1968, ZDF-Unternehmensarchiv, «Produktionsakte Der Reformator (Prod.-Nr. 06361/00119)».

63 Courrier de Bert BIEnIEk à Wolfgang Bruhn, 04.03.1968, ZDF-Unternehmensarchiv, «Produktionsakte Der Reformator (Prod.-Nr. 06361/00119)».

64 «Lutherexperte», ibid.

65 Courrier de Gottfried Edel à Bert Bieniek, 18.03.1968, ZDF-Unternehmensarchiv, «Produktionsakte Der Reformator (Prod.-Nr. 06361/00119)».

66 Courrier d'Elmar LoRey à Bert Bieniek, 05.04.1968, ZDF-Unternehmensarchiv, «Produktionsakte Der Reformator (Prod.-Nr. 06361/00119)».

67 La version initiale du scénario est consultable en archives: Günther SAwAtzki, Der Reformator. Martin Luther. Dokumentarspiel, ZDF-Unternehmensarchiv, «Produktionsakte Der Reformator (Prod.-Nr. 06361/00119)».
}

68 Courrier de Bert BIENIEK à Wolfgang BRUHn, 19.04.1968, ZDF-Unternehmensarchiv, "Produktionsakte Der Reformator (Prod.-Nr. 06361/00119)». 


\section{Entre mise à distance et réactualisation du mythe}

Si l'élaboration du scénario est marquée par une volonté didactique en rupture avec les représentations convenues du Réformateur, cette ambition se ressent également dans le résultat final à différents niveaux. L'élément le plus évident à cet égard est la rupture de l'«illusion du vrai» obtenue par la présence de deux types de séquences tout au long du film: à celles qui reconstituent des événements historiques par des acteurs en costume s'ajoutent des séquences de type documentaire, montrant à plusieurs reprises des gravures d'époque ou, dans la grande majorité des cas, l'auteur du scénario s'adresser à la caméra. Si les séquences documentaires visent la plupart du temps à apporter des informations qui permettent de mieux saisir le contenu des reconstitutions ou de faire le lien entre les différents événements montrés, leur intérêt didactique va bien au-delà. D’une part, ces séquences visent à familiariser le spectateur avec une certaine façon d'aborder l'histoire: le réalisateur Rudolf Jugert expliqua que l'alternance entre deux types de séquences constituait un «moyen d'éducation destiné à faire comprendre au public que nous construisons l'histoire à partir de faits» ${ }^{(69)}$, aspect sur lequel Sawatzki attire l'attention dès la séquence introductive du film.

D’autre part, les séquences documentaires révèlent la facticité des reconstitutions historiques, favorisant ainsi dans un esprit brechtien une approche analytique du cours des événements et du protagoniste plutôt qu'une immersion dans le récit ${ }^{(70)}$. Ce sont surtout les séquences consacrées aux propos de Sawatzki qui ont pour effet de briser l'illusion du vrai : lorsque Sawatzki se trouve face à la caméra, il est filmé à plusieurs reprises dans le décor utilisé auparavant pour les reconstitutions ${ }^{(71)}$.

Si Le Réformateur rompt sur le plan de la forme avec les représentations hagiographiques de Luther proposées à l'écran jusqu'alors, il en va de même au niveau de l'image du protagoniste qui se dégage du film, marquée par le souci de ramener à une taille humaine le héros surdimensionné par la tradition protestante et nationaliste. Premier élément «incontournable» présent dans Le Réformateur, l'affichage des 95 thèses est en même temps l'exemple le plus intéressant de ce refus de toute glorification hâtive. Certes, Sawatzki réhabilite cet épisode, dont la véracité avait été remise en question quelques années auparavant par l'historien de l'Église Erwin Iserloh ${ }^{(72)}$. Ce choix ne résulte cependant pas de la volonté de renouer sans distance critique avec la tradition héroïque héritée du XIX ${ }^{\mathrm{e}}$ siècle, mais d'une réflexion menée à partir des sources dont Sawatzki explique le détail: si l'affichage des thèses n'avait pas eu lieu, les contemporains de Luther n'auraient-ils pas contredit cette version des faits au moment où elle commença à se diffuser? Une source citée par Sawatzki rapporte que les thèses se diffusèrent en Allemagne en l'espace de quatorze jours; or, à partir de quel

69 «Erziehungsmittel dazu, um dem Publikum klarzumachen, daß wir aus den Fakten der Geschichte aufbauen», Horst G. FELDT, «Ein unromantisches und unheldisches Lutherbild. Ein in der Form neuartiges ZDF-Dokumentarspiel nach den Fakten (31. Oktober 1968) », epd Kirche und Film, 12.10.1968, cité d'après J. Horstmann (éd.), Martin Luther (note 7), p. 112-114, cit. p. 113.

70 H.-R. Schwa B, «Luther im deutschen Fernsehen» (note 7), p. 214-215; C. Hissnauer, «Geschichtsspiele im Fernsehen» (note 42), p. 302.

71 H.-R. Sсншав, «Luther im deutschen Fernsehen» (note 7), p. 215.

72 Erwin IserLoH, Luthers Thesenanschlag - Tatsache oder Legende?, Wiesbaden, Steiner, 1962. 
événement public ce laps de temps aurait-il pu être délimité, si ce n'est l'affichage des thèses? Certes contestables ${ }^{(73)}$, ces affirmations ont toutefois le mérite d'expliciter le raisonnement qui guida les choix de l'auteur du scénario. En outre, si Sawatzki plaide en faveur du caractère historique de l'événement, il n'en souligne pas moins le statut hypothétique par sa mise en scène à l'écran: la reconstitution ne montre pas Luther afficher le document au portail de l'église ${ }^{(74)}$ mais se contente de présenter la réaction du public.

Une fois commencé l'affrontement avec l'autorité ecclésiastique et séculière, Sawatzki se garde d'ériger le Réformateur en vaillant défenseur de la foi authentique et du redressement national. En effet, Le Réformateur rompt triplement avec la tradition hagiographique des films d'avant-guerre. Exploitant les possibilités offertes par le film parlant, Sawatzki ne se contente pas de présenter au public un florilège des citations les plus imposantes de Luther mais cherche à restituer au moyen des dialogues l'argumentation du moine pour rendre intelligible son cheminement intellectuel. Par ailleurs, Sawatzki n'hésite pas à pointer les éléments qui atténuent la grandeur du protagoniste. Enfin, l'acteur principal et le réalisateur écartent eux aussi les choix qui orienteraient le parcours et les propos de Luther vers une lecture apologétique.

Ainsi, la dispute avec le cardinal Cajetan (1518) est-elle moins l'occasion d'insister sur l'inflexibilité de Luther, humble et respectueux, saisi lors de son voyage à Augsbourg par des angoisses et des douleurs d'estomac ${ }^{(75)}$, que sur les raisons qui justifient son refus de révoquer. De même, la séquence consacrée à la dispute théologique de Leipzig entre Luther et Johannes Eck (1519) ne se contente pas de poser le constat que Luther nie l'autorité suprême du pape et des conciles en matière de dogme mais détaille pendant pas moins de six minutes les arguments par lesquels le Réformateur étaye sa position. Par un procédé similaire, la séquence de l'autodafé au cours duquel Luther brûle la bulle qui le menace d'excommunication (1520) ne se résume pas à la destruction du document, moment le plus spectaculaire aux yeux de la postérité, mais rappelle que ce geste fut accompagné par la mise au bûcher du droit canonique par les étudiants de Wittemberg ${ }^{(76)}$; un bref échange entre Luther et Melanchthon dévoile l'argumentaire qui sous-tend cette riposte de Luther à la menace romaine.

Enfin, la conception de la séquence consacrée à la diète de Worms (1521) est elle aussi guidée par la volonté de présenter une image adéquate et nuancée des motifs qui animèrent le Réformateur. Sawatzki ne tait pas que Luther, dérouté face à l'injonction qui lui fut adressée de révoquer ses écrits, demanda un temps de réflexion. Les propos qu'il tint lors de sa deuxième comparution devant l'empereur ${ }^{(77)}$ sont repris presque

73 L'historicité de l'affichage des thèses fait débat jusqu'à aujourd'hui; voir par exemple Volker LEPPIN, «Die Monumentalisierung Luthers. Warum vom Thesenanschlag erzählt wurde - und was davon zu erzählen ist", in: Joachim Oтт, Martin Treu (éd.), Luthers Thesenanschlag - Faktum oder Fiktion, Leipzig, Evangelische Verlagsanstalt, 2008, p. 69-92.

74 C'est ce que note H.-R. Schwaв, «Luther im deutschen Fernsehen» (note 7), p. 215.

75 Voir Martin Brecht, Martin Luther, t. 1 : Sein Weg zur Reformation 1483-1521, Stuttgart, Calwer Verlag, 1981, p. 242.

76 Voir ibid., p. 404

77 Deutsche Reichstagsakten unter Kaiser Karl V., t. 2, éd. par Adolf Wrede, Gotha, Friedrich Andreas Perthes, 1896, p. 551-555 et p. 575-582. 
intégralement par le scénario; les passages volontiers retenus par la tradition protestante et nationaliste sont ainsi insérés au sein d'un texte plus large qui, au-delà des pointes polémiques et des morceaux de bravoure, rend compte du raisonnement par lequel Luther déboucha sur la position qu'il adopta.

Outre le travail du scénariste Günther Sawatzki, celui de l'acteur Christian Rode et du réalisateur Rudolf Jugert contribue à faire naître une représentation de Luther qui s'écarte de la célébration de l'homme d'exception. Rode incarne un Luther qui,

Fig. 1 - Rudolf JUGERT, Le Réformateur, 1968. Photogrammes du film

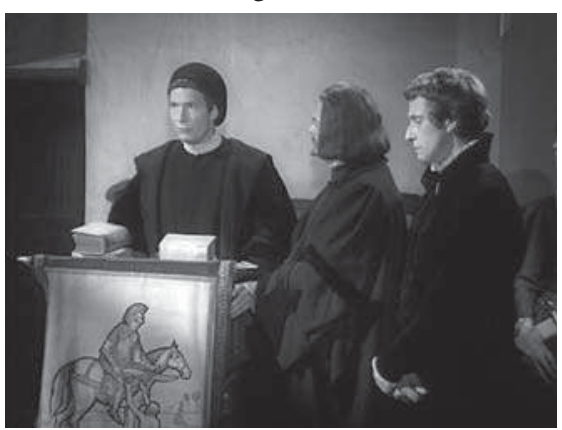

Luther face à Johannes Eck

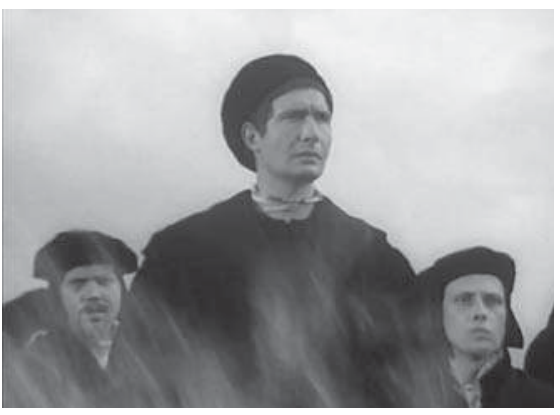

Luther désarçonné face à la réaction des fidèles lors de l'autodafé plus qu'un homme d'action intrépide, est un moine "guidé par l'intellect» ${ }^{(78)}$ : adoptant une gestuelle sobre, Rode se trouve la plupart $\mathrm{du}$ temps dans une position neutre, les mains jointes devant lui. Le courage et la détermination ne sont pas absents de son jeu, mais ils sont traduits de façon plus discrète au moyen du regard et de la voix. Les choix relatifs au cadrage tendent également à rompre avec une approche $\mathrm{du}$ personnage qui verserait dans le culte du grand homme: dans les séquences consacrées aux rencontres de Luther avec Cajetan et Eck, Jugert évite les échelles de plan qui centreraient l'attention du spectateur uniquement sur la prestation courageuse du Réformateur. La séquence de la dispute de Leipzig est certes ponctuée de plans rapprochés poitrine sur Luther; toutefois, les plans américains (voir fig. 1) et les demiensembles dominent, permettant de montrer un Luther entouré de ses adeptes et s'assurant de leur soutien. La séquence de l'autodafé privilégie le même type de plans; le seul plan rapproché centré sur le Réformateur vise à mettre en évidence non pas sa bravoure, mais sa surprise et sa désorientation face à la réaction des fidèles qui entonnent un Te Deum (voir fig. 1).

Incontestablement, Le Réformateur est marqué par une volonté de rupture avec la tradition hagiographique. Une analyse qui souhaite rendre justice au film ne peut toutefois pas s'arrêter là et doit insister sur un dernier point. Si le film déconstruit le héros national et le prophète venant rétablir la vraie foi, il ne nie pas pour autant la grandeur de Luther et n'en témoigne pas moins d'une sympathie certaine pour le penseur autonome qui défend sa conviction envers la hiérarchie. Luther n'est plus célébré comme le père de la nation ni de la foi protestante, mais il apparaît comme le modèle d'un esprit indépendant; la fonction fondatrice du mythe se trouve adaptée ainsi à la démocratie ouest-allemande de l'après-guerre. 
La représentation de Luther en esprit critique exemplaire passe par la mobilisation d'éléments ancrés dans la tradition, revus désormais à la lumière de la nouvelle signification attribuée au personnage. En effet, les étapes canoniques du parcours de Luther mettent l'accent sur la remise en question par le Réformateur d'une autorité prétendument incontestable: si les rencontres avec Cajetan, Eck et Charles Quint sont l'occasion de développer l'argumentation de Luther, c'est pour mettre en évidence le déploiement d'une pensée libérée de règles prétendument intouchables.

Pour ancrer dans les esprits le Luther «nouveau » qu'ils donnent à voir, Sawatzki et Jugert vont jusqu'à rompre avec les principes qui caractérisent la majeure partie du film en intégrant dans leur portrait du Réformateur des éléments légendaires ainsi que le rapprochement entre le Luther et le Christ, issus de la tradition contre laquelle l'auteur et le réalisateur affirmaient vouloir se dresser. Dans la séquence consacrée à la diète de Worms, le scénario renoue ainsi avec deux éléments traditionnels qui ne sont pas documentés de source fiable, soulignant par ce biais l'admirable intrépidité de Luther: on voit le capitaine de lansquenets Georg von Frundsberg saluer le courage du moine et Luther achever son discours par la fameuse formule apocryphe «Hier stehe ich $[\ldots]{ }^{(79)}$. Allant dans le sens du scénario de Sawatzki, les choix de Jugert privilégient dans la séquence de Worms les plans qui isolent Luther de son entourage: le plan rapproché poitrine sur Luther domine, complété à deux reprises par un gros plan (voir fig. 2). Renouant avec l'idée ancienne de la christiformitas du Réformateur, Sawatzki intègre au scénario le sermon sur l'excommunication prononcé par Luther en 1518 et variant le sermon sur la montagne ${ }^{(80)}$; Christian Rode est filmé en contre-plongée, choix qui va dans le sens de la transfiguration opérée par les

Fig. 2 - Rudolf JUGERT, Le Réformateur, 1968. Photogramme du film

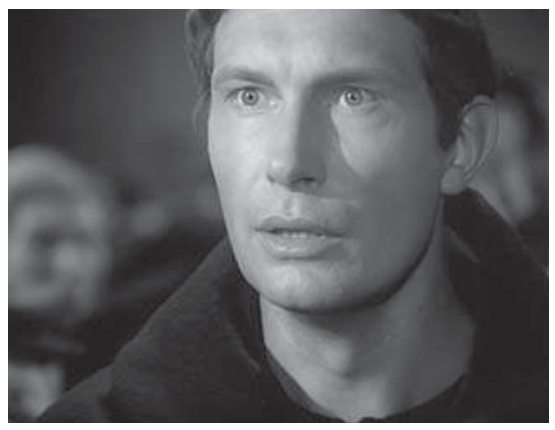

Luther à la diète de Worms paroles de l'acteur.

\section{Conclusion : quelle réception?}

La réception de la pièce documentaire Le Réformateur est en ceci digne d'intérêt qu'elle témoigne de mutations sociales dans le domaine religieux et confessionnel qui rendirent possible d'une part l'acceptation d'une image nuancée du père du protestantisme et se manifestèrent de l'autre par une certaine baisse de l'intérêt pour les sujets à caractère religieux. Auprès des téléspectateurs, la production n'atteignit qu'un audimat

79 Sur le premier point, voir Henrike Holsing, Luther - Gottesmann und Nationalheld. Sein Image in der deutschen Historienmalerei des 19. Jahrhunderts, thèse de doctorat dirigée par Hans Ost, université de Cologne, 2004, kups.ub.uni-koeln.de/2132/2/diss_text_holsing.pdf, p. 468; sur le deuxième, voir H. SCHILling, Martin Luther (note 24), p. 223.

80 Martin Luther, Sermo de virtute excommunicationis, in: ID., Werke. Kritische Gesamtausgabe (Weimarer Ausgabe). Schriften, t. 1, éd. par Joachim Karl Friedrich KNAAKE, Weimar, Hermann Böhlau, 1883 , p. 634-643, voir p. 643. 
de $16 \%$ lors de sa diffusion le 31 octobre 1968 à 20 h00 ${ }^{(81)}$, soit à l'occasion de la «Fête de la Réforme» (Reformationsfest) et aux heures de grande écoute. L'institut de sondage Infratest jugea les résultats atteints par l'ensemble des programmes diffusés par les deux premières chaînes allemandes ce soir-là "assez faibles " ${ }^{(82)}$ et les imputa au caractère religieux et «sérieux» ${ }^{(83)}$ de la thématique; l'appartenance confessionnelle ne joua un rôle qu'auprès de «certains » ${ }^{(84)}$ téléspectateurs catholiques qui ne se sentirent pas concernés par le programme proposé. Dans le cas précis de l'émission commentée ici, la faiblesse de l'audimat fut sans doute due également à l'aspect didactique du film que la presse jugea à plusieurs reprises trop scolaire, maladroit et rébarbatif ${ }^{(85)}$. Toutefois, Le Réformateur fut évalué positivement par les téléspectateurs qui virent l'émission ${ }^{(86)}$.

Dans la presse, l'écho critique fut dans l'ensemble favorable. On loua « une image de Luther libérée de sa composante romantique et héroïque» ${ }^{(87)}$; appréciant la démarche de Sawatzki, le même critique constata avec satisfaction que la mise en scène des événements fut systématiquement «ramenée à son point de départ historique, à savoir le document ${ }^{(88)}$. L'acteur Christian Rode contribuait à l'effet d'ensemble par une «grande retenue dans sa gestuelle et sa mimique» ${ }^{(89)}$. Attentif au dialogue œcuménique, on reconnut le rôle positif que la production pouvait jouer à cet égard ${ }^{(90)}$. Sensible à la fonction exemplaire que Sawatzki et Jugert avaient attribuée à un Luther incarnant la révolte d'une conscience contre l'autorité, on pointa les rapports entre le parcours de Luther et l'époque présente: "L'émancipation intellectuelle du chrétien, la liberté de la décision prise face à sa conscience, ne s'agit-il pas là de thèmes qui sont toujours d'actualité aujourd'hui?» ${ }^{(91)}$, se demanda un critique de la Westdeutsche Rundschau, tandis qu'un autre suggérait des «parallèles avec les événements de l'année 1968 » ${ }^{(92)}$. Seule une voix s'éleva pour réclamer une image de Luther plus monumentale ${ }^{(93)}$; plus

81 Infratest-Berichte, t. 41-53/1/196 (07.10.68-05.01.69), p. 19, ZDF-Unternehmensarchiv.

82 «recht niedrig», ibid.

83 «ernst[-]», ibid.

84 «manche», ibid.

85 Voir (KWE), «Wörter», Allgemeine Zeitung, 02.11.1968; (csf), « Der Reformator », Die Welt, 02.11.1968, ZDF-Unternehmensarchiv, «Pressespiegel Okt.-Dez. $1968 »$.

86 Infratest-Berichte, t. 41-53/1/196 (07.10.68-05.01.69), p. 18, ZDF-Unternehmensarchiv.

87 H. FELDT, «Ein unromantisches und unheldisches Lutherbild» (note 69).

88 «zurückgeführt auf seinen historischen Ausgang, das Dokument», (ok), "Der Reformator. FernsehDokumentarspiel über Martin Luther», Evangelischer Film-Beobachter, no 514 (1968), cité d'après J. Horstmann (éd.), Martin Luther (note 7), p. 111.

89 «starke Zurückhaltung in Gestik und Mimik», ibid.

90 Eva Patzig, «Was wir sahen: Fromme Juden, streitbare Christen», Mannheimer Morgen, 02.11.1968; (HB), «Der Reformator. Zum Reformationsfest. Dokumentarspiel über Martin Luther», Augsburger Allgemeine, 31.10.1968, ZDF-Unternehmensarchiv, «Pressespiegel Okt.-Dez. 1968». Voir également J. Horstmann (éd.), Martin Luther (note 7), p. 112.

91 «Die Mündigkeit des Christen, die Freiheit der Gewissensentscheidung, sind diese Themen nicht auch heute erneut aktuell?», (Anon.), «Das große theologische Welttheater», Westdeutsche Rundschau, 02.11.1968, ZDF-Unternehmensarchiv, «Pressespiegel Okt.-Dez. 1968 ».

92 «Parallelen zu den Ereignissen im Jahr 1968», (HB), «Der Reformator» (note 90).

$93(\mathrm{Ru})$, «Aber es war nicht Martin Luther. Ein Reformationsspiel im Zweiten Fernsehen », Hannoversche Allgemeine Zeitung, 02.11.1968, ZDF-Unternehmensarchiv, «Pressespiegel Okt.-Dez. 1968». 
représentatif du regard distancé que l'on était prêt désormais à poser sur le Réformateur, un critique estima que Luther restait encore trop proche d'un "personnage nazaréen idéalisé» ${ }^{(94)}$, travers qui aurait pu être évité si l’on avait intégré les travaux récents menés par la psychanalyse ${ }^{(95)}$.

La production de 1968 eut-elle à long terme une influence sur l'image télévisuelle et cinématographique de Martin Luther? On est en droit d'en douter. Certes, la mythisation du Réformateur en sujet émancipé fondateur de l'époque moderne persiste dans certains films récents, mais il s'agit là d'un discours trop répandu pour pouvoir être directement imputé à la "pièce documentaire» écrite par Sawatzki. La juxtaposition de séquences reconstituées et de séquences documentaires se retrouve dans les docufictions allemandes des années 2000, mais elle y a pour effet d'authentifier la fiction bien plus que de briser l'illusion du vrai ${ }^{(96)}$. La retenue et le souci de la nuance dans la représentation du Réformateur enfin ne nous semble pas être, dans l'ensemble, la principale caractéristique des films récents; valable pour le fameux Luther, co-production allemande, américaine et britannique du réalisateur Eric Till sortie en salle en $2003^{(97)}$, ce constat s'applique également à plusieurs téléfilms allemands des années 2000 qui, s'ils ne retombent pas dans l'hagiographie aveugle, tendent à ériger Luther en figure fédératrice d'un pays récemment réunifié( ${ }^{(98)}$.

Indice à la fois d'une prise de distance vis-à-vis du mythe luthérien et d'un réinvestissement de celui-ci, document d'époque qui permet de croiser sources écrites et sources visuelles, Le Réformateur illustre de toute évidence l'intérêt que doit revêtir le film de télévision pour l'étude de la réception de Luther dans l'espace germanophone. On peut espérer que cette attention s'étendra jusqu'aux productions les plus récentes, qui ont vu le jour dans le cadre du dernier jubilé, et celles qui sont peut-être à venir pour le $500^{\mathrm{e}}$ anniversaire de la diète de Worms en 2021.

\section{Résumé}

Dans le cadre des jubilés luthériens récemment passés ou en cours de préparation - les 500 ans de la Réforme en 2017, les 500 ans de la diète de Worms en 2021 -, la mémoire de Martin Luther en Allemagne a suscité un nouvel intérêt. Parmi les supports qui la véhiculent et l'entretiennent, le film de cinéma et de télévision détient une place de choix. C'est à cet aspect que se consacre le présent article, centré sur une production ouest-allemande diffusée sur le petit écran en 1968 et très peu étudiée par la recherche jusqu'ici. Il s'agit de montrer que le film est marqué par un double mouvement: d'une

94 «nazarenisch idealisierte Gestalt», Klaus Hamburger, «Illustrierter Religionsunterricht. Günther Sawatzki: “Der Reformator”, Dokumentarspiel über Martin Luther», Funk-Korrespondenz, 07.11.1968, cité d'après J. Horstmann (éd.), Martin Luther (note 7), p. 115. Également cité chez E. WiPfLER, «Vom deutschnationalen Titan» (note 7 ), p. 35, note $n^{\circ} 34$.

95 K. Hamburger, «Illustrierter Religionsunterricht» (note 94), p. 115.

96 E. Wipfler, «Filmstar Martin Luther» (note 7), p. 99; F. SCHLie, Luther à l'écran (note 4), p. 524-526.

97 E. Wipfler, Martin Luther in Motion Pictures (note 4), p. 60-62.

98 E. Wipfler, «Filmstar Martin Luther» (note 7), p. 98-99; F. Schlie, Luther à l'écran (note 4), p. 489-535. 
part, la volonté de rompre avec les représentations hagiographiques et convenues qui, nourries par les milieux protestants et nationalistes, avaient eu cours jusqu'en 1945; de l'autre, la tentative d'investir le mythe luthérien sous un jour nouveau, susceptible d'aider à la construction d'une société démocratique et pluraliste. Le travail s'achève par une étude de la réception dont bénéficia le film auprès des téléspectateurs et de la presse.

\section{Zusammenfassung}

Im Rahmen des kürzlich begangenen und des bevorstehenden Lutherjubiläums - dem 500. Jahrestag der 95 Thesen 2017, dem 500. Jahrestag des Wormser Reichstags 2021 - ist die Frage des Luthergedächtnisses in Deutschland erneut in den Fokus gerückt. Unter den Medien, die dieses Gedächtnis verbreiten und unterhalten, hat der publikumswirksame Fernseh- und Kinofilm einen besonderen Platz inne. Diesem Aspekt widmet sich der vorliegende Aufsatz, indem er sich mit einer westdeutschen Fernsehproduktion aus dem Jahre 1968 beschäftigt, die bislang von der Forschung kaum beachtet wurde. Es soll gezeigt werden, dass der Film von einer doppelten Tendenz geprägt ist. Einerseits will er mit dem hagiographischen und überkommenen, von nationalistischen und protestantischen Milieus genährten Lutherbild brechen, das bis 1945 in Umlauf war. Andererseits will er den Luthermythos neu gestalten, indem er ihn zum Fundament einer demokratischen und pluralistischen Gesellschaft erhebt. Der Aufsatz schließt mit einer Analyse der Rezeption, die dem Film seitens der Fernsehzuschauer und der Presse zuteilwurde. 